\title{
X-Ray Phase Nano-tomography by FZP-based X-Ray Microscopy Combined with Talbot Interferometry
}

$\underline{\text { Yanlin Wu}}^{{ }^{*}}$, Hidekazu Takano ${ }^{2}$, Karol Vegso ${ }^{2}$, Masato Hoshino², Koichi Matsuo ${ }^{3}$, Atsushi Momose ${ }^{1,2}$

1. Institute of Multidisciplinary Research for Advanced Materials, Tohoku University, Sendai, Japan.

2. Japan Synchrotron Radiation Research Institute, Sayo, Japan.

3. Keio University School of Medicine, Tokyo, Japan.

*yanlin.wu.a1@tohoku.ac.jp

A 100-fold full-field X-ray microscope using a Fresnel zone plate (FZP) in combination with a Talbot interferometer for phase tomography was constructed at beamline 37XU, SPring-8 to visualize the morphology of mouse bone specimens with a sub-micron spatial resolution and keeping with $300 \mu \mathrm{m}$ wide-field of view [1]. However, there was a problem that bone specimens shrunk occasionally due to synchrotron radiation irradiation during CT scans. In this work, a higher-efficiency FZP for 9 keV X-rays has been employed to observe bone samples having soft tissue structures with reduced radiation damage.

In this work, we constructed a 74.1-fold X-ray microscope with a FZP (ANT HZP-500 $\mu$ m-100nm) of a diameter of $500 \mu \mathrm{m}$ and a diffraction efficiency of $30.2 \%$ at $9 \mathrm{keV}$ in combination with a Talbot interferometer (see Table 1) which was constructed at beamline 37XU as well. The Talbot interferometer consisting of a $\pi / 2$ phase grating $2.39 \mu \mathrm{m}$ in pitch and an absorption grating $2.4 \mu \mathrm{m}$ in pitch was set in front of the image detector whose effective pixel size was $13.65 \mu \mathrm{m}$. Phase tomography was performed using the continuous fringe-scanning method [2].

By evaluation, the diffraction efficiency of the new FZP performed almost as designed; that is, the total radiation dosage to bone specimens has been reduced by four times than the previous experiment [1] with total exposure time of 0.5 hours. Figure 1 shows a cross-sectional phase tomogram of bone specimen (malleus of mouse, 14 days after birth) obtained in the previous experiment. The shrinkage of bone specimen was observed as motion artefacts, as indicated by yellow arrows especially at soft tissues inside the bone. In contrast, the soft tissue and bone has been clearly observed in this study without motion artefacts, as shown in Figure 2.

In conclusion, we have improved the image quality of phase tomograms by using a FZP of a higher diffraction efficiency and a large diameter for the X-ray microscope combined with a Talbot interferometer. It is expected that the presented approach for X-ray phase nano-tomography is effective for various applications including biological studies [3].

References:

[1]. A. Momose et al, SPIE Proc. 10391 (2017) 103910Y.

[2]. S. Kibayashi et al, AIP Conf. Proc., 1466 (2012), 261.

[3]. The experiments were performed in project 2016B1147, 2017A1288, 2017B1361 at BL37XU SPring8, Japan. This study was financially supported by the ETATO-Momose quantum beam phase imaging project (Grant Number JPMJER1403) of Japan Science and Technology Agency (JST), Japan. 
Table 1. Parameters of ATN/FZP--SiC86/416 (NTT Advanced Technology Corporation [NTT-AT], Japan) FZP used in ref. [1] and HZP (Hard X-ray Fresnel zone plates) -500 $\mu$ m-100nm FZP (Applied Nanotools Inc [ANT], Canada) employed in this study.

\begin{tabular}{|c|c|c|}
\hline Parameter & ATN/FZP-SiC86/416 & HZP-500um-100nm \\
\hline Diameter & $416 \mu \mathrm{m}$ & $500 \mu \mathrm{m}$ \\
\hline Outermost zone width & $86 \mathrm{~nm}$ & $100 \mathrm{~nm}$ \\
\hline Zone thickness & 700 nm (Ta) & 1469 nm (Au) \\
\hline Membrane thickness & $2 \mu \mathrm{m}(\mathrm{SiC})$ & 100 nm (SiN) \\
\hline Focal length@9 keV & $261 \mathrm{~mm}$ & $365 \mathrm{~mm}$ \\
\hline $1^{\text {st }}$ order diffraction efficiency @9 keV & $8.6 \%$ & $30.2 \%$ \\
\hline $0^{\text {st }}$ order diffraction efficiency @ 9 keV & $72.2 \%$ & $7.6 \%$ \\
\hline
\end{tabular}

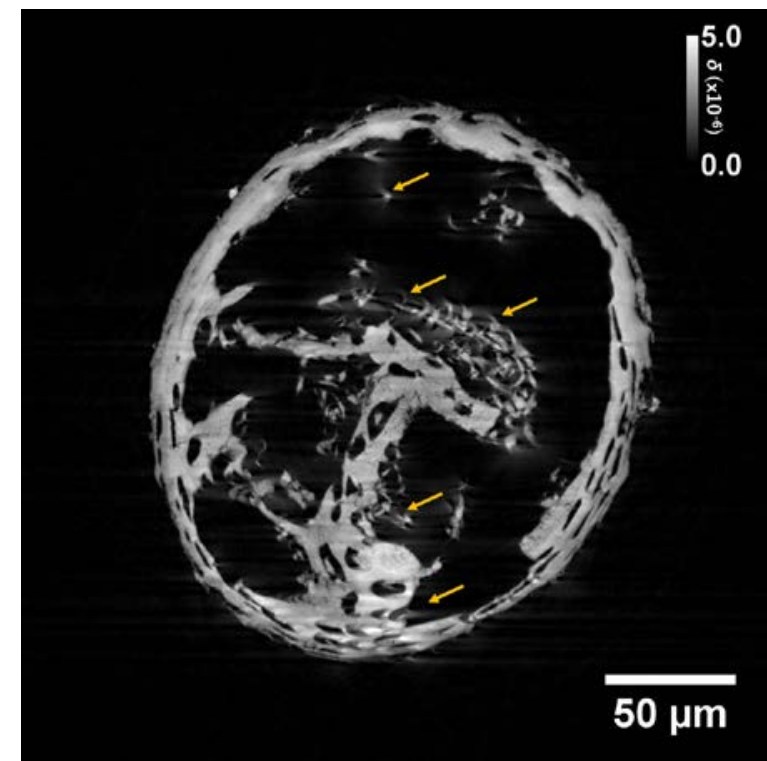

Figure. 1. A phase tomogram (cross-section) of a bone specimen (malleus of mouse) obtained by the previous [1].

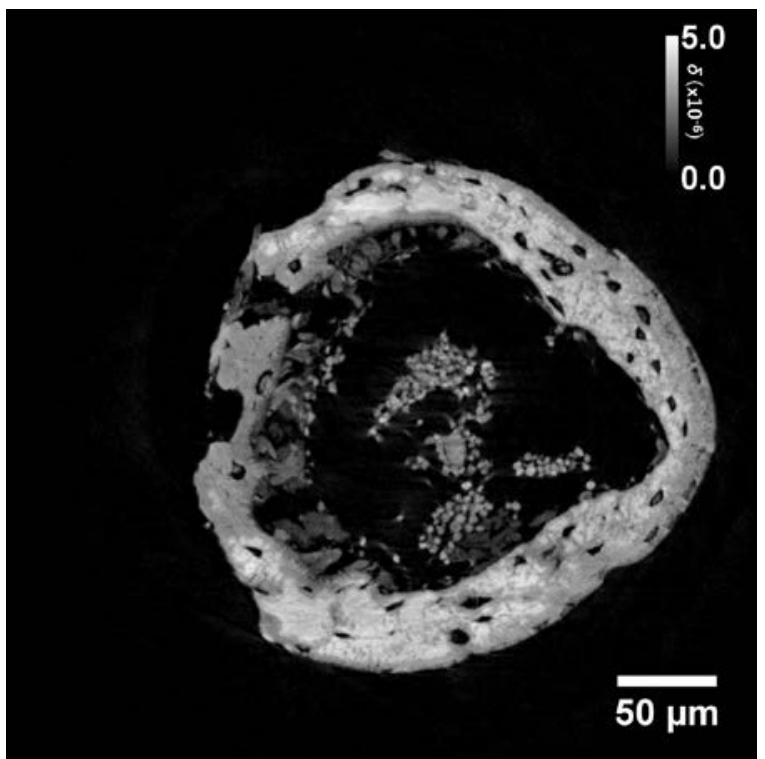

Figure. 2. A phase tomogram (cross-section) of a bone specimen obtained in this work. 